\title{
Fluorescence Studies on Self-association of Biodegradable Pyrene-labeled Poly(L-lysine) Grafted with Poly(caprolactone)
}

\author{
Kenji Kimura and Yasumasa Fukushima* \\ Department of Biological Applied Chemistry, Graduate School of Engineering, \\ Toyo University, 2100 Kujirai, Kawagoe, Saitama 350-8585, Japan \\ *E-mail: fukusima_m@toyo.jp
}

\begin{abstract}
The biodegradable amphiphilic graft copolymer (PolyLysPy-PCL) containing hydrophilic pyrene end-labeled poly(L-lysine) (PolyLysPy) backbone and hydrophobic polycaprolactone (PCL) side chains was synthesized by the amide condensation of PolyLysPy and PCL. PolyLysPy was prepared from ring-opening polymerization of $N$ - $\varepsilon$-benzyloxycarbonyl-L-lysine $N$-carboxyanhydride (Lys(Z)-NCA) initiated by 1-pyrenemethylamine and followed by removing the side-chain protective groups. PCL was prepared from the enzymatic ring-opening polymerization of $\varepsilon$-caprolactone by a porcine pancreas lipase. The structures and number-average molecular weights $(\mathrm{Mn})$ of the graft copolymer and its precursors were confirmed and investigated by the ${ }^{1} \mathrm{H}$ NMR measurement. The self-assembly of the graft copolymer in water/DMSO mixture solution was examined by fluorescent signals assigned to the pyrene moiety. The increase in the grafting percent of PCL to a PolyLysPy polymer backbone can facilitate the self-assembly by the hydrophobic interaction of associated PCL grafts.
\end{abstract}

Keywords : biodegradable, amphiphilic graft polymer, polylysine, polycaprolactone

\section{Introduction}

Over the past few decades, amphiphilic copolymers have attracted considerable interest owing to their unique self-assembly and phase behavior properties in solution and their numerous practical applications in surfactants, flocculating agents, drug delivery systems, and biomedical field [1-3]. Amphiphilic copolymers composed of hydrophilic and hydrophobic segments can form micelles with a hydrophobic compact inner core and a hydrophilic swollen outer shell in aqueous media $[4,5]$. The hydrophobic core serves as a carrier environment for hydrophobic drugs, and the hydrophilic outer shell stabilizes the micelles in solution $[6,7]$. Although most of the amphiphilic copolymers studied for drug delivery system are made of block copolymers, graft copolymers have gained increasing attention due to the adjustable backbone and side chains $[8,9]$. In particular, graft copolymers containing a great quantity of side chains chemically attached onto a linear backbone possess confined and compact structures compared with block copolymers [10, 11]. However, relatively few works have been done on amphiphllic graft copolymers despite the numerous advantages of possessing intriguing structures in solution and providing integrations of considerable functionality onto the polymer backbone, especially for graft copolymers with a hydrophilic backbone and hydrophobic side chains $[12,13]$.

Currently, amphiphilic biodegradable polymers became a class of the most attractive biomaterials because of their wide applications in pharmaceutical and biomedical fields [14-16]. Among the variety of known biodegradable polymers, aliphatic polyesters such as poly ( $\varepsilon$-caprolactone) (PCL) have been widely used as the hydrophobic segment of the amphiphilic copolymers because of controlled degradability, excellent biocompatibility, and flexible mechanical properties $[17,18]$. One procedure for the production of well-controlled PCL is the 
ring-opening polymerization (ROP) of cyclic $\varepsilon$-caprolactone $(\boldsymbol{\varepsilon}-\mathbf{C L})$ monomer in the presence of catalysts such as stannous octanoate [19]. Recently, the enzymatic polymerization of $\boldsymbol{\varepsilon}$-CL has been extensively studied due to the many advantages including mild reaction conditions, high selectivity, and the use of nontoxic catalysts [20]. This green synthetic process can thus be suitable for biodegradable and biocompatible polymeric materials.

Poly( $\alpha$-amino acid)s are well known as very important synthetic biomaterials with low immunogenicity, good biocompatibility and excellent mechanical properties suitable for various biomedical applications [21]. Due to their polypeptide backbone, they have the potential to be degraded in biological environments. Poly ( $\alpha$-amino acid)s were readily synthesized by the ring-opening polymerization (ROP) of $\alpha$-amino acid $N$-carboxyanhydrides (NCAs) with an amino compound. Poly(L-lysine) is also the hydrophilic, biocompatible, and biodegradable polymer of L-lysine which is a proteinogenic amino acid. Poly(L-lysine), has reactive amino groups on the side chain, can be modified by a condensation reaction with functional compounds or introduction of other polymeric components to graft structures. Amphiphilic copolymers containing poly(L-lysine) have been studied widely because a condensation reaction is a simple and convenient way to functionalize poly(L-lysine) directly [22-24]. However, there have been a few reports on the synthesis of the amphiphilic biodegradable copolymer consisting of poly(L-lysine) and poly( $\varepsilon$-caprolactone) $[25,26]$.

Fluorescence techniques are used as useful tools to investigate the solution properties of amphiphilic copolymers with high sensitivity and selectivity which can determine the critical micelle concentration and the aggregation number of the hydrophobic moiety [27, 28]. Pyrene compounds have proved to be a particularly valuable fluorescent probe due to their advantageous characteristics such as high fluorescence quantum yield, long fluorescence lifetime, and strong excimer emission [29, 30]. Pyrene end-functionalized polymers have been reported recently to prove the polymer dynamics at the molecular level although pyrene has been usually used as an external probe for studying the self-assembly of vesicles and micelles [31-33].

In the present study, a biodegradable amphiphilic graft copolymer consisting in hydrophilic polylysine as a backbone and hydrophobic poly( $\varepsilon$-caprolactone) as a branch was prepared via a facile and efficient pathway as shown in scheme 1. Poly(L-lysine) with amino functional groups was synthesized by the ring-opening polymerization (ROP) of a lysine $N$-carboxyanhydride (NCA) with pyrene bearing a primary amino group. Poly( $\varepsilon$-caprolactone) was then conjugated to the backbone by an amide coupling method. The structure of the copolymer was characterized by ${ }^{1} \mathrm{H}$ NMR and FT-IR. The characterization of self-association and micellization for the pyrene-end-labelled copolymer was studied by a fluorometric measurement

\section{Experimental}

2.1 Materials

All chemicals were obtained from commercial suppliers and used without further purification.

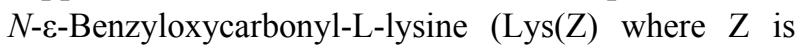
benzyloxycarbonyl) was purchased from Peptide Institute Inc. Lipase from porcine pancreas (PPL) was obtained from Sigma-Aldrich Chemical Co. Methanesulfonic acid (MSA) and diethyl phosphorocyanidate (DEPC) were purchased Tokyo Chemical Industries Co., Ltd.

\subsection{Measurements}

NMR spectra were obtained at $400 \mathrm{MHz}$ on a JEOL JNM-EX400 with TMS as internal standard at room temperature. Fluorescence spectra were recorded on a JASCO FP-750 spectrofluorometer. All fluorescence measurements were carried out in in DMSO: $\mathrm{H}_{2} \mathrm{O}(50: 50, \mathrm{v} / \mathrm{v})$ upon excitation at 340 $\mathrm{nm}$ (polymer concentration: $0.1 \mathrm{mg} / \mathrm{mL}$ ).

\subsection{Synthesis}

\subsubsection{Lys(Z)-NCA}

Lys(Z)-NCA was prepared referring to the method reported in the literature [34]. $\operatorname{Lys}(\mathbf{Z})(18.0$ $\mathrm{g}, 64.2 \mathrm{mmol}$ ) was suspended in anhydrous THF $(720 \mathrm{~mL})$ with dry nitrogen gas, and triphosgene ( $8.66 \mathrm{~g}, 29.2 \mathrm{mmol})$ was added. The suspension was stirred at $55^{\circ} \mathrm{C}, 1 \mathrm{~h}$ later the reaction mixture turned to clear, after $2 \mathrm{~h}$ the reaction finished. The clear solution was allowed to cool to room temperature, washed with water and aqueous $\mathrm{NaHCO}_{3}$, concentrated to $120 \mathrm{~mL}$ in reduced pressure, and precipitated with hexane $(720 \mathrm{~mL})$. Lys(Z)-NCA was recrystallized twice from hexane and ethyl acetate and dried in vacuo to afford a white solid (18.4 g, 93.7\%). 


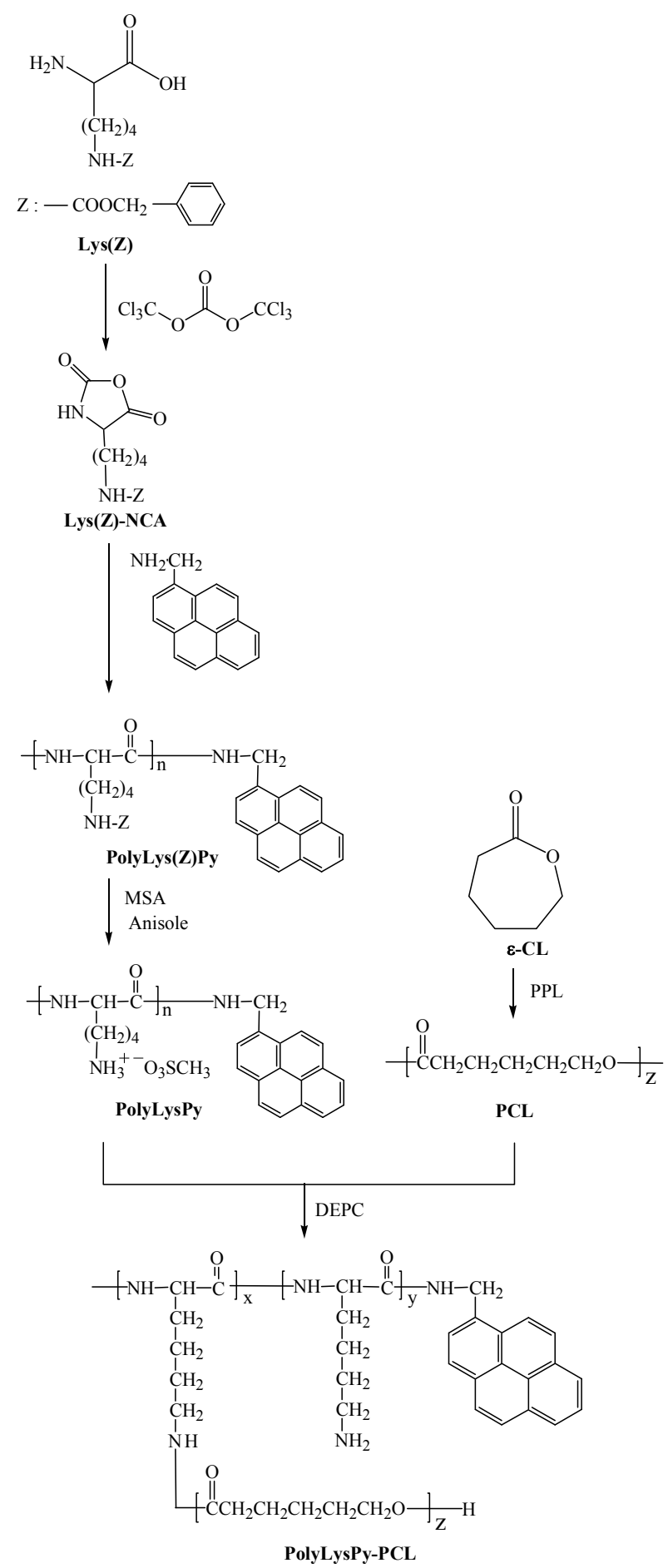

Scheme 1 Synthetic route of an amphiphilic copolymer

\subsubsection{PolyLysPy}

To a solution of 1-pyrenemethylamine hydrogen chloride $(2.00 \mathrm{~g}, 7.48 \mathrm{mmol})$ in DMSO $(80 \mathrm{~mL})$ was added $\mathrm{NaOH}(0.30 \mathrm{~g}, 7.50 \mathrm{mmol})$ in methanol $(2 \mathrm{~mL})$ and the mixture solution was stirred at room temperature for $24 \mathrm{~h}$. The solution was evaporated and the obtained solid was washed with water $(200 \mathrm{~mL})$ and diethyl ether $(100 \mathrm{~mL})$, dried in vacuo. To a solution of $\operatorname{Lys}(\mathbf{Z})-\mathbf{N C A}(2.50 \mathrm{~g}$, $8.15 \mathrm{mmol})$ in anhydrous THF $(20 \mathrm{~mL})$ was added 1-pyrenemethylamine $(0.13 \mathrm{~g}, 0.55 \mathrm{mmol})$ in DMSO $(0.04 \mathrm{~mL})$ under a dry nitrogen atmosphere. After stirring at $0{ }^{\circ} \mathrm{C}$ for $3 \mathrm{~h}$ and at room temperature for $90 \mathrm{~h}$, the reaction mixture was poured into diethyl ether $(50 \mathrm{~mL})$ and the precipitate was filtered and dried under reduced pressure to afford a solid. After a treatment of the solid with MSA (42 $\mathrm{mL})$ in the presence of anisole $(4.2 \mathrm{~mL})$ at room temperature for $3 \mathrm{~h}$, the reaction mixture was poured into diethyl ether $(600 \mathrm{~mL})$. The resulting precipitate was filtered, washed with acetone, dried in vacuo to yield a pale yellow solid $(1.6 \mathrm{~g}, 71 \%)$.

\subsubsection{PCL}

A suspension of $\varepsilon$-CL $(6.18 \mathrm{~g}, 54.2 \mathrm{mmol})$ and PPL $(1.30 \mathrm{~g})$ in heptane $(5 \mathrm{~mL})$ was stirred at $70{ }^{\circ} \mathrm{C}$ for $72 \mathrm{~h}$. The reaction mixture was filtered for removal of heptane, and the precipitate was washed with distilled water $(700 \mathrm{~mL})$. The crude precipitate was dissolved in $250 \mathrm{~mL}$ of chloroform and precipitated in $250 \mathrm{~mL}$ hexane. The purified product was filtered and dried in vacuo.

\subsubsection{PolyLysPy-PCL}

A solution PolyLysPy $(1.02$ g) and triethylamine $(1.38 \mathrm{~g})$ in DMSO $(50 \mathrm{~mL})$ was added to PCL ( 0.223 or $0.446 \mathrm{mg}$ ) in chloroform at the molar ratio of PCL to $\mathrm{ENH}_{2}$ of PolyLysPy, $1 / 10$ or $1 / 5$, respectively. To the mixture solution was added DEPC (1.13 g), after stirring at room temperature for $24 \mathrm{~h}$ and evaporation of DMSO and chloroform, the precipitate was washed with distilled water. After a solution of the crude precipitate in chloroform was filtered, the filtrate was poured in toluene. The precipitate was filtered and dried in vacuo to give the desired PolyLysPy-PCL graft copolymers.

\section{Results and discussion}

Oligopeptides were usually synthesized by stepwise solid-phase or liquid-phase synthesis, while poly $(\alpha$-amino acid $)$ s were easily prepared by ring-opening polymerizations of $N$-carboxyanhydride (NCA) of $\alpha$-amino acid. Amino acids at their side chain with reactive functional groups which can interfere with NCA synthesis or polymerization need to be protected with suitable groups that can be removed after completion of the polymerization. L-Lysine $(\operatorname{Lys}(\mathbf{Z}))$ with the $\varepsilon$-amino group protected with a benzyloxycarbonyl (Z) group was used for poly(L-lysine) synthesis. The protecting group can 
be selectively eliminated under strong acidic conditions after completion of the polymerization.

According to the Fuchs-Farthing method [34], $\operatorname{Lys}(Z)-N C A$ was synthesized by reacting $\operatorname{Lys}(Z)$ with triphosgene in anhydrous THF under dry nitrogen atmosphere in an approximately quantitative yield of $94 \%$. Because the protonation of the evolved hydrogen chloride as a by-product to the unreacted amino group of an amino acid is caused by reducing its nucleophilicity and solubility, the nitrogen gas was sparged under the reaction in order to discharge the hydrogen chloride, resulting in an excellent yield. The reaction solution, was initially turbid owing to the poor solubility of $\mathbf{L y s}(\mathbf{Z})$ in THF, which became gradually clear by the conversion to $\mathbf{L y s}(\mathbf{Z})-\mathbf{N C A}$. After reaction completion, the reaction solution washed with cold water and $\mathrm{NaHCO}_{3}$ aqueous solution to remove acidic contaminants. $\operatorname{Lys}(\mathbf{Z})-N C A$ was precipitated with hexane and purified by repeated recrystallization from hexane and ethyl acetate, resulting in a white crystal.

The polymerization of NCA can be initiated by a variety of nucleophiles such as water, alcohols, and amines. Among the NCA polymerization initiators, primary amines can be quantitatively incorporated at the $\mathrm{C}$ terminus of the polypeptide chain due to undergoing a nucleophilic addition to the C-5 carbonyl group of NCA [35]. In order to incorporate a pyrene moiety into the terminus of poly(L-lysine) as a fluorescent probe, pyrene with a primary amine was used as a initiator of NCA polymerization. Prior to use as a polymerization initiator, 1-pyrenemethylamine hydrogen chloride was treated with $\mathrm{NaOH}$ methanol solution for the conversion from the ammonium salt to its primary free amino group. Protected poly(L-lysine) was prepared by performing the ring opening polymerization of $\mathbf{L y s}(\mathbf{Z})$-NCA in THF/DMSO mixture under a dry nitrogen atmosphere using 1-pyrenemethylamine as a initiator and a fluorescent probe (monomer to initiator ratio $=15$ ). The polymerization was performed at $0{ }^{\circ} \mathrm{C}$ for first $3 \mathrm{~h}$ to suppress the side reactions at the chain ends, which commonly lead to in-ring structures and chain-end termination [36]. After polymerization, pyrene-labeled polypeptide (PolyLys(Z)Py) was precipitated with diethyl ether, filtered, and dried in vacuo. PolyLys(Z)Py was analyzed via ${ }^{1} \mathrm{H}$ NMR in order to identify the polypeptide structure, to confirm the incorporation of the pyrene moiety, and to determine the number-average molecular weight. As shown in Figure 1, the ${ }^{1} \mathrm{H}$ NMR spectrum of PolyLys(Z)Py in DMSO- $\mathrm{d}_{6}$ presents the typical resonance signals attributed to $\operatorname{PolyLys}(Z)$ protons and a multiplet signal centered at $8.2 \mathrm{ppm}$ attributed to the resonance of the pyrene ring protons. From Figure 1, the degree of polymerization (DP) of PolyPly(Z)Py was calculated to be 14.6 by a comparison of the integral ratio of the signals at $8.2 \mathrm{ppm}$ corresponded to pyrene aromatic protons to that at $5.0 \mathrm{ppm}$ corresponded to $\mathrm{CH}_{2}$ protons of the benzyl group. The number-average molecular weight (Mn) of PolyPly(Z)Py was determined to be 4,056 by the ${ }^{1} \mathrm{H}$ NMR spectrum. The expected molecular weight from the monomer to initiator ratio and measured one are in relatively good agreement.

Treatment of PolyLys(Z)Py with methanesulfonic acid in the presence of anisole was performed in order to remove the benzyloxycarbonyl (Z) groups. Although deprotected pyrene-labeled poly(L-lysine) (PolyLysPy) was soluble in DMSO, it showed better solubility in water. The removal of the protecting group was characterized by ${ }^{1} \mathrm{H}$ NMR in $\mathrm{D}_{2} \mathrm{O}$ (Figure 2). The complete disappearance of the signals at 7.3 and 5.0 corresponded to the benzyl protons confirmed the formation of PolyLysPy from Figure 2. The number-average molecular weight (Mn) of PolyPlyPy was determined to be 2,100 by the ${ }^{1} \mathrm{H}$ NMR spectrum.

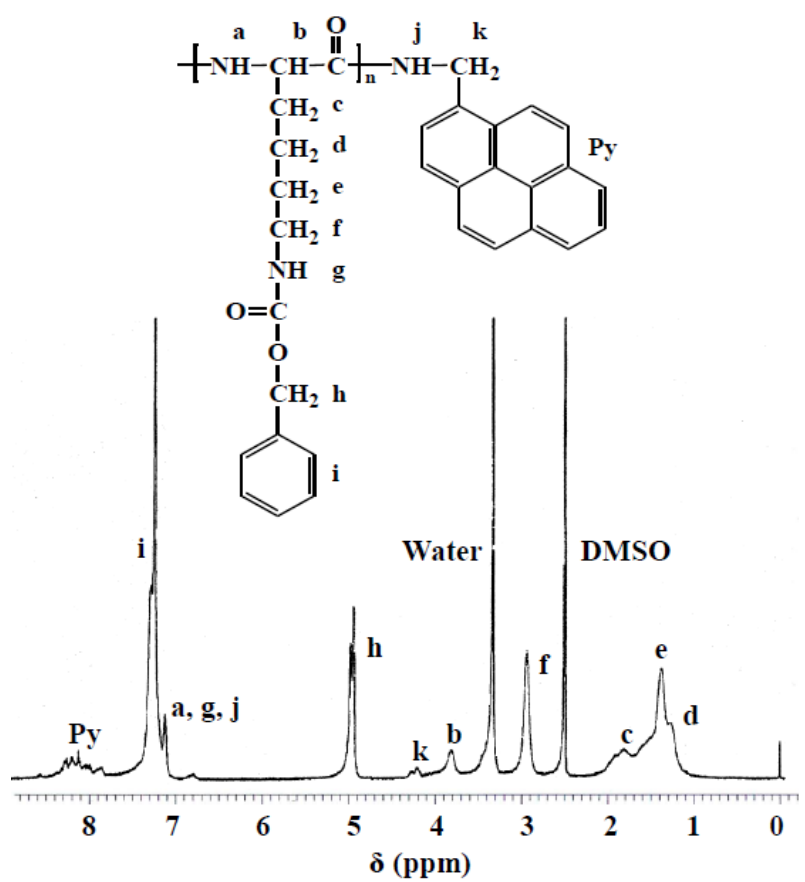

Figure 1. ${ }^{1} \mathrm{H}$ NMR spectrum of PolyLys(Z)Py in DMSO-d ${ }_{6}$. 


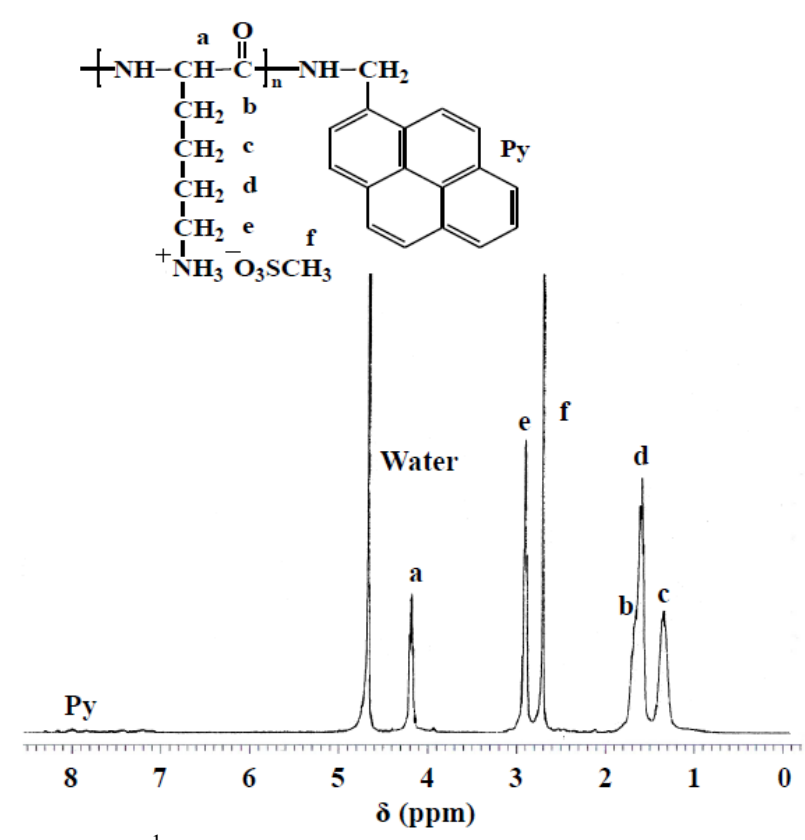

Figure 2. ${ }^{1} \mathrm{H}$ NMR spectrum of PolyLysPy in $\mathrm{D}_{2} \mathrm{O}$.

The synthesis of polycaprolactone (PCL) was carried out via enzymatic ring-opening polymerization using lipase from porcine pancreas as the catalyst. PCL was soluble in $\mathrm{CHCl}_{3}$, but insoluble in water and DMSO. DP of PCL was calculated to be 7.9 by the comparison of the integral ratio of the signals at $4.1 \mathrm{ppm}$ corresponded to the $\mathrm{CH}_{2}$ protons adjacent to the oxygen of the ester group and $3.7 \mathrm{ppm}$ corresponded to the $\mathrm{CH}_{2}$ protons adjacent to the end hydroxyl group in the ${ }^{1} \mathrm{H}$ NMR spectrum shown in Figure 3. The number-average molecular weight (Mn) of PCL was determined to be about 919 by the ${ }^{1} \mathrm{H}$ NMR spectrum.
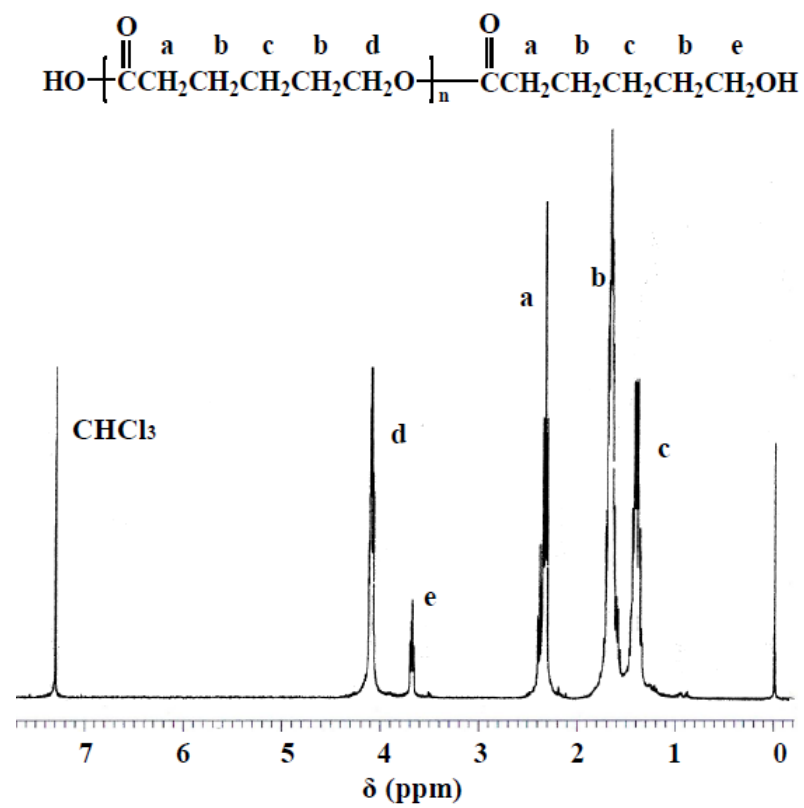

Figure 3. ${ }^{1} \mathrm{H}$ NMR spectrum of $\mathbf{P C L}$ in $\mathrm{CDCl}_{3}$.

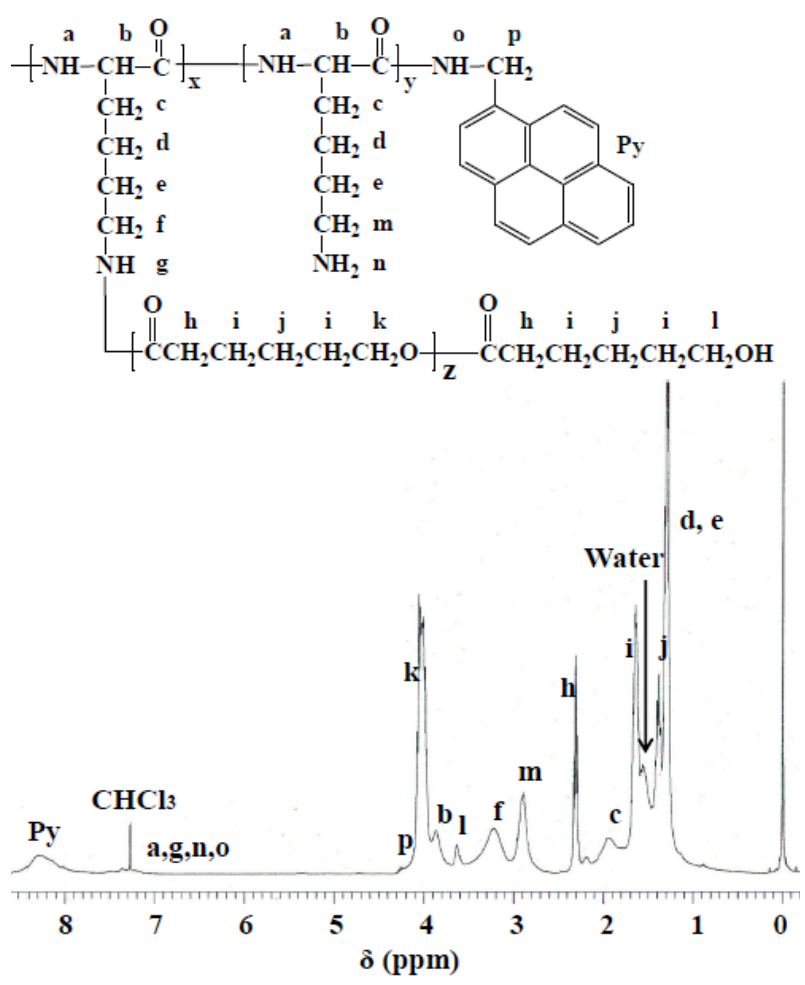

Figure 4. ${ }^{1} \mathrm{H}$ NMR spectrum of PolyLysPy-PCL6 in $\mathrm{CDCl}_{3}$.

PolyLysPy grafted with PCL was synthesized through the condensation reaction of the amino group of PolyLysPy with the end-carboxyl group of PCL using DEPC as an amide coupling reagent. PolyLysPy-PCL was purified by removal of unreacted PolyLysPy and PCL from the reaction mixture with water and toluene, respectively. PolyLysPy-PCL could be dissolved in DMSO and chloroform, but it was insoluble in water and toluene. Two samples of PolyLysPy-PCL with different grafting percentages were synthesized by changes in the feed molar ratio of PCL to the amino groups of PolyLysPy The grafting percentage of PolyLysPy-PCL at $10 \mathrm{~mol} \%$ feed molar ratio was calculated to be $6.0 \mathrm{~mol} \%$ by the comparison of the integral ratio of the signals at $8.3 \mathrm{ppm}$ corresponded to the pyrene protons and $2.3 \mathrm{ppm}$ corresponded to the $\mathrm{CH}_{2}$ protons adjacent to the carbonyl group of PCL in ${ }^{1} \mathrm{H}-\mathrm{NMR}$ spectrum shown in Figure 4 (PolyLysPy-PCL6). For $20 \mathrm{~mol} \%$ feed molar ratio, the grafting percentage can be determined to be $9.0 \mathrm{~mol} \%$ (PolyLysPy-PCL9). The low grafting percentages compared with the feed molar ratios could be attributed to macromolecular effects.

The amphiphilic graft polymers, PolyLysPy-PCL6 and PolyLysPy-PCL9 presented a good solubility in common solvents 
such as DMSO, DMF, and chloroform. Given the limited solubility of PolyLysPy-PCL6 and PolyLysPy-PCL9 in water due to the hydrophobic segment PCL, their hydrophobic association experiment in a mixture of DMSO: $\mathrm{H}_{2} \mathrm{O}$ (50:50, $\mathrm{v} / \mathrm{v}$ ) by fluorescence spectroscopy was performed. As shown in Figure 5, the fluorescence spectra derived from a pyrene covalently bonded to the polymers were observed. For PolyLysPy and PolyLysPy-PCL6, three maxima at 382, 401, and $423 \mathrm{~nm}$, characteristic of the monomer emission, are present. On the other hand, for PolyLysPy-PCL9, a new structureless peak centered at $448 \mathrm{~nm}$ appears, which can be attributed to excimer emission. The presence of excimer emission indicates interaction of pyrene groups in the excited state through intermolecular excimer formation by the pyrene terminals. When the PCL content contained in the amphiphilic polymers is increased, the self-association would be caused by a PCL aggregation due to the increased hydrophobicity.

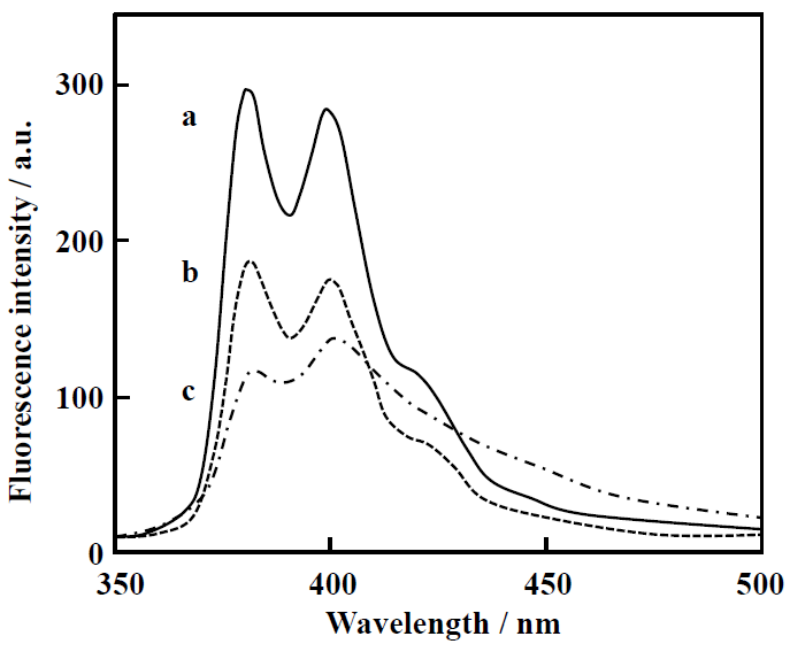

Figure 5. Fluorescent spectra of (a)PolyLysPy, (b)PolyLysPy-PCL6, and (c)PolyLysPy-PCL9 in DMSO: $\mathrm{H}_{2} \mathrm{O}(50: 50, \mathrm{v} / \mathrm{v})$ upon excitation at $340 \mathrm{~nm}$ (concentration: $0.1 \mathrm{mg} / \mathrm{mL}$ ).

The intensity ratio $\left(I_{1} / I_{3}\right)$ of the first band to the third band of pyrene emission was used to monitor the local environment of the pyrene molecules in surfactants or amphiphilic polymers [37, 38]. When pyrene is in a more nonpolar environment, the $I_{1} / I_{3}$ value is lower [39]. Pyrene is preferentially solubilized into the interior of the hydrophobic regions in polar media. This change in the microenvironment of pyrene leads to the change such as $I_{1} / I_{3}$ value in its emission spectrum. The $I_{1} / I_{3}$ value was calculated by taking the ratio of the intensity at $382 \mathrm{~nm}$ to that at $392 \mathrm{~nm}$. The $I_{1} / I_{3}$ values are $1.39,1.38$, and 1.07 for PolyLysPy, PolyLysPy-PCL6, and PolyLysPy-PCL9, respectively. The $I_{1} / I_{3}$ value decreases with an increase in the PCL content of the amphiphilic polymers. In particular, for PolyLysPy-PCL9, the $I_{1} / I_{3}$ value exhibited a drastic decrease because the pyrene would be selectively located in the aggregated area of the PCL segments by the hydrophobic interaction. This result indicates that PolyLysPy-PCL9 forms the micellar structure which is composed of PCL as a core and PolyLys as a shell.

\section{Conclusion}

In conclusion, two biodegradable amphiphilic graft copolymers, PolyLysPy-PCL6 and PolyLysPy-PCL9, consisting of hydrophilic pyrene end-labeled poly(L-lysine) backbone with the different content of the PCL side chain were synthesized. The graft polymers demonstrated the ability to form the self-association by the hydrophobically intermolecular interaction of PCL segment. This polymer could be a potentially useful for polymeric amphiphilic materials with low environmental loading.

\section{References}

1. A.O. Moughton, M.A. Hillmyer, T.P. Lodge, Macromolecules, 45 (2012) 2.

2. C.J. Hawker, K.L.Wooley, Science, 309 (2005) 1200 .

3. C. Helmut, Macromol. Rapid Commun. 22 (2001) 219.

4. G. Riess, Prog. Polym. Sci., 28 (2003) 1107.

5. G. Carrot, J. Hilborn, D.M. Knauss, Polymer, 38 (1997) 6401.

6. R. Savic, A. Eisenberg, D. Maysinger, J. Drug Target., 14 (2006) 343.

7. N. Rapoport, Prog. Polym. Sci., 32 (2007) 962.

8. H.Q. Xie, D. Xie, Prog. Polym. Sci., 24 (1999) 275.

9. M.G. McKee, S. Unal, G.L. Wilkes, T.E. Long,. Prog. Polym. Sci., 30 (2005) 507.

10. H.I. Lee, J. Pietrasik, S.S. Sheiko, K. Matyjaszewski, Prog. Polym. Sci., 35 (2010) 24.

11. P. Li, J. Zhu, P. Sunintaboon, F.W. Harris, Langmuir, 18 (2002) 8641.

12. J.H. Jeong, H.S. Kang, S.R. Yang, J.D. Kim, (2002). Polymer, 44 (2002) 583.

13. K. Breitenkamp, T. Emrick, J. Am. Chem. Soc., 125 (2003) 12070.

14. H.Y. Tian, Z.H. Tang, X.S. Chen, X.B. Jing, Prog. Polym. Sci., 37 (2012) 237.

15. H. Guan, Z. Xie, P. Zhang, C. Deng, X. Chen, X. Jing, Biomacromolecules, 6 (2005) 1954. 
16. J. Sun, X. Chen, T. Lu, S. Liu, H. Tian, Z. Guo, X. Jing, Langmuir, 24 (2008) 10099.

17. A.L. Chen, H.C. Ni, L.F. Wang, J.S. Chen, Biomacromolecules, 9 (2008) 2447.

18. M. Zhang, H. Liu, W. Shao, K. Miao, Y. Zhao, Macromolecules, 46 (2013) 1325.

19. L. Liu, Y. Wang, X. Shen, Y. Fang, Biopolymers, 78 (2005) 163.

20. I.K. Varma, A.C. Albertsson, R. Rajkhowa, R.K. Srivastava, Prog Polym Sci., 30 (2005) 949.

21. T. Fukuoka, H. Uyama, S. Kobayashi, Biomacromolecules, 5 (2004) 977.

22. Y.H. Choi, F. Liu, J.S. Kim, Y.K. Choi, J.S. Park, S.W. Kim, J. Controlled Release, 54 (1998) 39.

23. C. Deng, X.S. Chen, H.J. Yu, J. Sun, T.C. Lu, X.B. Jing, Polymer, 48 (2007) 139.

24. C.W. Zhao, X.L. Zhuang, C.L. He, X.S. Chen, X.B. Jing, Macromol. Rapid Commun., 29 (2008) 1810.

25. N. Xu, F.S. Du, Z.C. Li, J. Polym. Sci. Part A Polym. Chem., 45 (2007) 1889.

26. X. He, L. Zhong, K. Wang, S. Luo, M. Xie, J. Appl. Polym. Sci., 117 (2010) 302.

27. A. Yekta, B. Xu, J. Duhamel, H. Adiwidjaja, M.A. Winnik, Macromolecules, 28 (1995) 956.
28. Y. Koyama, M. Umehara, A. Mizuno, M. Itaba, T. Yasukouchi, K. Natsume, Bioconjugate Chem., 7 (1996) 298.

29. K. Nishikawa, A. Yekta, H.H. Pham, M.A. Winnik, Langmuir, 14 (1998) 7119.

30. M.R. Pokhrel, S.H. Bossmann, J. Phys. Chem. $B, 104$ (2000) 2215.

31. B. Haldar, A. Mallick, N. Chattopadhyay, J. Mol. Liq., 115 (2004) 113.

32. H. Siu, J. Duhamel, J. Phys. Chem. B, 116 (2012) 1226.

33. M.A. Winnik, S.M. Bystryak, Z. Liu, J. Siddiqui, Macromolecules, 31 (1998) 6855.

34. H.W. Daly, D. Poche, Tetrahedron Lett., 29 (1998) 5859.

35. T.J. Deming, J. Polym. Sci. Part A Polym. Chem., 38 (2000) 3011.

36. G.J.M. Habraken, K.H.R.M. Wilsens, C.E. Koning, A. Heise, Polym. Chem., 2 (2011) 1322.

37. K. Kalyanasundaram, J. Thomas, J. Am. Chem. Soc., 99 (1977) 2039.

38. A. Nakajima, Photochem. Photobiol., 25 (1977) 593.

39. K.P. Ananthapadmanabhan, E.D. Goddard, N.J. Turro, P.L. Kuo, Langmuir, 1 (1985) 352. 